example, those dealing with the solution to equations, nomograms and probability, will inevitably be more widely read because they deal with requirements which arise more widely in practice. In fact, although this may not bring out its full value, "Biomathematics" is likely to be used chiefly as a reference book to be dipped into as the need arises, and its use in this way will be all the easier for the glossary of symbols provided at the beginning. The need for such a source of mathematical reference is more widespread than even biologists themselves probably feel.

So long a mathematical book can scarcely fail to be expensive, and it is perhaps an inevitable mis fortune that at $80 \mathrm{~s}$. the price of "Biomathematics" will limit its sale. It is to be hoped, nevertheless, that younger biologists will at least have ready access to Dr. Smith's book in their laboratories, and will be encouraged to use it. For, given the oppor. tunity, it can offer much-needed help. K. MAther

\section{PRINCIPLES OF ANALYTICAL INORGANIC CHEMISTRY}

Grundlagen der analytischen Chemie und der Chemie in wässerigen Systemen

Von Prof. F. Seel. Pp. 348. (Weinheim/Bergstr. Verlag Chemie, 1955.) 29 D. marks.

T HIS book deals in five chapters with the separation of metal ions by precipitation techniques, reactions in molten salts, various volumetric titrations and electrolytic separation methods. In twenty chapters problems of more general interest are set out: namely, definitions and fundamental theories, mass action law, solubilities, theories of precipitation, colloid chemical effects, formation of complexes, proton transfer reactions, acid-base equilibria, ion exchangers and oxidation-reduction processes.

Students who read this book are liable to get an unbalanced view, because the usefulness of the mass action law in deafing with heterogeneous systems is over-emphasized and because many important topics are omitted ; for example, the relative contributions of $\Delta S$ and $\Delta H$ to $\Delta F$ of a chemical equilibrium, or an appropriate treatment of reaction kinetics and mechanism. Furthermore, there is no coherent story, the presentation being confusing and not consistent. Thus, the mass action law is derived, $a b$ initio, in Chapter 2, while the derivation of Nernst's formula, connecting electromotive force with ionic activities, is only very briefly indicated in an appendix, although it is extensively used in the last ten chapters. There are statements such as "reactions with positive $p K$ values use up energy and reactions with negative $p K$ values yield energy" ( $p K$ is the negative logarithm of the equilibrium coefficient), or "the energy of dissociation of certain complexes must be derived from a precipitation reaction". In other sections reactions are classed as "inhibited", although it is not suggested that an inhibitor influences the kinetics, the failure to reach equilibrium within a short time being merely due to the energy or entropy of activation. Again, the term "physical" solubility is introduced, implying that this is different from other solubilities, but there is no definition; and many numerical values of $p K$ are listed, in the text and in four tables of the appendix, without specifying the temperature.
Advanced research workers and university teachers, on the other hand, should not be unduly disturbed by these and similar shortcomings ; but this class of readers, if interested in analytical chemistry, will miss a discussion of colorimetric, flame photometric, chromatographic and polarographic methods, and representative literature references.

It must be pointed out, however, that five out of the twenty-five chapters are rather enjoyable to read, provided they are considered as separate short monographs. These chapters contain instructive numerical calculations and some useful information not given in conventional text-books of analytical chemistry.

ALBERT WASSERMANN

\section{LENS THEORY}

Theory of Lenses

By E. W. H. Selwyn. (Monographs for Students.) Pp. 62. (London : Institute of Physics, 1955.) 5s.

RY the theory of lenses, Mr. E. W. H. Selwyn means a discussion, in terms of physical optics, of the behaviour of real lenses. This book is intended for students preparing for the Higher National Certificate in Applied Physics, and is one of a series bridging the gap between the elementary text-books and the academic and technical treatises. Its outlook is original and refreshing, and the author's long experience of both lenses and learners has enabled him to deal handsomely by both. His aim, he says, is that the reader shall not be too much surprised by some of the things he may notice when using optical devices or trying to make them in the laboratory. One feels that the student who has fully mastered the contents of these few pages will really understand what he is doing in such circumstances, for this is an excellent introduction with a very practical approach.

After an initial discussion of the wave theory of light, in which the beginner's difficulties with Huygens's construction are admirably resolved, the author traces the effect of a refracting surface on a wave-front diverging from a point, considering in turn the image formed by a spherical refracted wavefront corresponding to paraxial conditions, and the distortion of this wave-front due to aberrations. A fair knowledge of co-ordinate geometry is here assumed; but the mathematical treatment is clear and straightforward. Next, the terms in the expression for the distorted wave-front corresponding to the second-order Seidel aberrations are identified, and their effects on the image and their correction are explained. The last chapter deals with correction for colour, high-order aberrations, and the balancing procedure of the lens designer in achieving some cancellation of one aberration by a higher-order aberration of the same kind.

The book ends with a selection of problems, some simple exercises on the book-work and some particularly searching ones. None, says Mr. Selwyn, can be solved merely by substituting numerical values in a correctly remembered formula - a comment which the late Prof. John Perry might have expanded into many vehement paragraphs, and which conveys all too delicately a warning against the student's greatest temptation. But the reader who has followed Mr. Selwyn's treatment will naturally be thinking in terms of physics rather than of formulæ, so that perhaps even so gentle a reminder may prove superfluous.

G. R. NOAKES 